\title{
Dynamical qubit controlling via pseudo-supersymmetry of two-level systems
}

\author{
Boris F. Samsonov and V. V. Shamshutdinova \\ Tomsk State University, 36 Lenin Avenue, 634050 Tomsk, Russia
}

\begin{abstract}
For a flux qubit considered as a two-level system, for which a hidden polynomial pseudo-supersymmetry was previously discovered, we propose a special time-dependent external control field. We show that for a qubit placed in this field there exists a critical value of tunnel frequency. When the tunnel frequency is close enough to its critical value, the external field frequency may be tuned in a way to keep the probability to detect a definite direction of the current circulating in a Josephson-junction circuit above $1 / 2$ during a desirable time interval. We also show that such a behavior is not much affected by a sufficiently small dissipation.
\end{abstract}

PACS numbers: 03.67.Lx, 03.75.Lm, 85.25.Am 


\section{Introduction}

In the past few years superconducting circuits based on Josephson tunnel junction have attracted much attention both from theoretical and experimental viewpoints as possible candidates for the implementation of quantum computer (see, e.g. Refs. $[1,2,3])$. Usually they represent a small Josephson-junction circuit, called a Cooperpair box, which consists of a small superconducting electrode connected to a reservoir via a Josephson junction [2]. For a flux qubit the circuit with a very small inductance containing three Josephson junctions is described (in appropriate units) by the following two-level Hamiltonian [3]:

$$
H_{q}=-\Delta \sigma_{x}-\varepsilon(t) \sigma_{z} .
$$

Here $\Delta$ is the tunnel frequency, and $\varepsilon(t)$ is a time-dependent field (bias) which is controlled by an externally applied flux. Although in general $\Delta$ is a function of $\varepsilon$, it varies on the scale of the single junction plasma frequency, which is much above the typical energy range at which the qubit is operated [4]. We thus can assume $\Delta$ to be constant for the purpose of this paper. Solving the Schrödinger equation with Hamiltonian (1),

$$
i \dot{\Psi}(t)=H_{q} \Psi, \quad \Psi=\left(\psi_{1}, \psi_{2}\right)^{T}
$$

(superscript " $T$ " means the transposition and the dot over a symbol means the derivative with respect to time), we obtain the probability of a definite direction of the current circulating in the ring, i.e. $P^{\downarrow}=\left|\psi_{1}\right|^{2}$ is the probability of the clockwise direction of the current and $P^{\uparrow}=\left|\psi_{2}\right|^{2}$ is the probability of the opposite current direction.

One of the most important problem in quantum computation is connected with the possibility of controlling the state of an array of qubits. Typically the simplest two-qubit operations are generated by interplay of the coupling between qubits and local fields. Much theoretical attention has been recently paid to studying the controllable coupling between qubits of different types (see Ref. [5] and references therein). Recently it has been shown [6] that in the simplest and the most important from engineering viewpoint case of an "always on" and fixed coupling, a two-qubit Hamiltonian may be decoupled and the control problem is, in particular, reduced to finding the evolution of a one qubit placed in a time-dependent external control field. This observation shows an additional importance of controlling a one qubit state. This is the main subject we devote the present paper.

Usually, the probabilities, as functions of time, show an oscillating behavior (cf. famous Rabi oscillations, see e.g. Ref. [7]). But for some specific external fields this character may be changed drastically thus showing a possibility to control the qubit state. Up to now such a possibility is known to be mostly related to oscillating external fields [8].

Recently [9] it was proposed to consider a two-level Schrödinger equation as a Dirac equation with a non-Hermitian Hamiltonian where time plays the role of a spatial variable. This possibility revealed a hidden pseudo-supersymmetry which may be associated with a two-level system [10] and lead to discovering new time-dependent external fields where a two-level system admits solutions in terms of elementary functions. An advantage of analytic solutions is the possibility of a careful analysis of their properties which may reveal unexpected peculiarities [11]. In this paper we apply these results to show the possibility of controlling the qubit state with an external field of a special configuration. We show that there exists a critical value of the tunnel 
frequency $\Delta$. While the tunnel frequency approaches this critical value, the probability $P^{\downarrow}(t)$ oscillates around a value exceeding $1 / 2$ with a decreasing amplitude and after the critical value is reached it becomes a function monotonously increasing up to limiting value equal $3 / 4$. Then using the property that this special excitation regime is, in fact, a limiting case of a more general oscillating external field, we demonstrate that one can control a definite direction of the current in the ring (i.e. the qubit state) during a desirable time interval. Finally we also show that such a behavior is not much affected by the presence of a reasonably small dissipation featuring open quantum systems. We start with reminding the reader main constructions leading to polynomial pseudo-supersymmetry in two-level systems which is done in the next section.

\section{Polynomial pseudo-supersymmetry of two-level systems}

Similar to the conventional supersymmetry in quantum mechanics (for recent developments see a special issue of 2004 J. Phys. A: Math. Gen. 34 (43)), pseudosupersymmetry is based on intertwining and factorization relations. However, in this case Hamiltonians are non-Hermitian and a specific automorphism, that defines pseudo-adjoint operators, should be involved $[12,13]$. In particular if $A$ is a linear operator and $\eta$ is linear, Hermitian, invertible operator then

$$
A^{\sharp}=\eta^{-1} A^{+} \eta,
$$

where ' + ' sign denotes the usual (e.g. Laplace) adjoint operation, by definition is the pseudo-adjoint of $A$ with respect to $\eta$. The operation of formal (Laplace) conjugation obeys the standard rules $(A B)^{\dagger}=B^{\dagger} A^{\dagger},(d / d t)^{\dagger}=-d / d t$ and corresponds to the transposition of a matrix accompanied by the complex conjugation of its elements. Operator $B$ is said to be pseudo-Hermitian with respect to $\eta$ if $B^{\sharp}=B$, i.e.

$$
B=\eta^{-1} B^{+} \eta \text {. }
$$

Basic properties of pseudo-Hermitian operators are discussed in detail in [13].

Recently [10] it was observed that a polynomial pseudo-supersymmetry may be associated with a two-level system interacting with a classical (i.e. not quantized) electromagnetic field. The method is based on the possibility to rewrite the Schrödinger equation (2) that governs the evolution of the system in the form of a one-dimensional stationary Dirac equation

$$
h_{0} \Psi=E \Psi,
$$

where the time plays the role of a spatial variable and the Dirac Hamiltonian

$$
h_{0}=i \sigma_{x} \frac{d}{d t}+V_{0}(t), \quad V_{0}(t)=i \sigma_{y} \varepsilon_{0}(t), \quad E=\Delta
$$

is non-Hermitian, with the subsequent application of the well-developed intertwining operators technique [14]. Function $\varepsilon(t)$ plays the role of a "potential".

It is easy to see that $h_{0}(6)$ is pseudo-Hermitian with respect to $\eta=\sigma_{x}$. The next ingredient of the method is based on the existence for any real-valued function $\varepsilon_{0}(t)$ such real-valued function $\varepsilon_{1}(t)$ and operator $L$ (intertwiner) that

$$
L h_{0}=h_{1} L
$$

where $h_{1}=i \sigma_{x} d / d t+V_{1}(t)$ with $V_{1}=i \sigma_{y} \varepsilon_{1}(t)$. The pseudo-Hermiticity of $h_{0}$ results in the following factrorizations

$$
\eta L^{+} \eta L=h_{0}^{2}-\Lambda^{2}, \quad L \eta L^{+} \eta=h_{1}^{2}-\Lambda^{2} .
$$


The constant matrix $\Lambda=\operatorname{diag}(\lambda,-\lambda)$ in (8) is called the (matrix) factorization constant ( $\lambda$ is also called the factorization constant). Formulas (8) present a generalization of the factorization properties of transformation operators that take place in the case of Hermitian one-component Hamiltonians [15]. If now we introduce matrix operators (in block-matrix forms)

$$
H=\left(\begin{array}{cc}
h_{0} & 0 \\
0 & h_{1}
\end{array}\right), \quad Q_{1}=\left(\begin{array}{cc}
0 & 0 \\
L & 0
\end{array}\right), \quad Q_{2}=\left(\begin{array}{cc}
0 & J L^{+} J \\
0 & 0
\end{array}\right)
$$

then the intertwining (7) and factorization (8) relations may be rewritten as the following set of commutation and anti-commutation relations between these operators:

$Q_{1,2}^{2}=0, \quad H Q_{1,2}=Q_{1,2} H, \quad Q_{1} Q_{2}+Q_{2} Q_{1}=H^{2}-\operatorname{diag}(\Lambda, \Lambda)$

which indicate on the simplest quadratic pseudo-superlagebra. Note that the subsequent application of this technique leads to a more general polynomial pseudosupersymmetry [10]. On the other hand if we start from $V_{0}$ with known solutions to the Dirac equation (5) then solutions of the same equation with $V_{1}$ can be obtained by applying $L$ to the previous solutions. In this way new exactly solvable two-level potentials are obtained [10] which we use in the next section to demonstrate the possibility of the dynamical qubit controlling.

\section{Dynamical qubit controlling}

Consider first the case when the external control field $\varepsilon=\varepsilon_{1}(t)$ changes in the following way:

$$
\varepsilon_{1}(t)=-\varepsilon_{0}+\frac{4 \varepsilon_{0}}{1+4 \varepsilon_{0}^{2} t^{2}} .
$$

Parameter $\varepsilon_{0}$ gives us the possibility to choose a suitable time scale since after rescaling $\tau=2 \varepsilon_{0} t$, and redefining parameter $\Delta, \Delta=2 \varepsilon_{0} \delta$, we obtain the Schrödinger equation with the Hamiltonian

$$
H=-\delta \sigma_{x}-\epsilon(\tau) \sigma_{z}, \quad \epsilon(\tau)=\epsilon_{1}(\tau)=-\frac{1}{2}+\frac{2}{1+\tau^{2}}
$$

for which exact analytic solutions are known [10]. Therefore imposing the initial condition $P^{\downarrow}(0)=0$ we can write down an explicit expression for the probability $P^{\downarrow}(\tau)$ :

$$
\begin{aligned}
& P_{1}^{\downarrow}(\tau)=\frac{\left(\theta^{2}-1\right)\left(\theta^{2}+4\right)}{2 \theta^{4}} \frac{\tau^{2}}{1+\tau^{2}}+\frac{\left(\theta^{2}-1\right)\left(\theta^{2}-4\right)}{2 \theta^{6}\left(1+\tau^{2}\right)} \\
& \times\left[\theta^{2}-4-\left(\theta^{2}-4+\theta^{2} \tau^{2}\right) \cos \theta \tau+4 \theta \tau \sin \theta \tau\right]
\end{aligned}
$$

where we have introduced $\theta=\sqrt{1+4 \delta^{2}}$. From here it is clearly seen that $P_{1}^{\downarrow}(\tau)$ is an oscillating function provided $\theta \neq 2(\delta \neq \sqrt{3} / 2)$. For $\theta=2(\delta=\sqrt{3} / 2)$ equation (13) yields

$$
P_{1}^{\downarrow}(\tau)=\frac{3}{4} \frac{\tau^{2}}{1+\tau^{2}}
$$

which is a function monotonously increasing from zero at $\tau=0$ till the value $3 / 4$ for $\tau \gg 1$ (solid (black) line in Fig. 1). Since $\delta$ differs from $\Delta$ only by the scaling factor $2 \varepsilon_{0}$ we will call $\delta$ tunnel frequency as well. We note a decrease of the oscillation amplitude when $\delta$ approaches its critical value equal $\sqrt{3} / 2$. This is why for $\delta$ close 


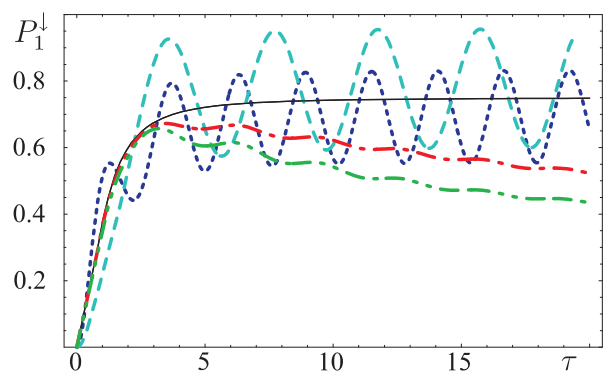

Figure 1. (Color online) Evolution of $P_{1}^{\downarrow}$ probability at $\delta=\sqrt{3} / 2$ solid, dotdashed and double-dot-dashed (black, red and green) lines, at dephasing $\left(\Gamma_{\varphi}\right)$ and relaxation $\left(\Gamma_{r}\right)$ rates: $\Gamma_{r}=\Gamma_{\varphi}=0.05$ dot-dashed (red) line and $\Gamma_{r}=\Gamma_{\varphi}=0.1$ double-dot-dashed (green) line; $\delta=\sqrt{3} / 2 \pm 0.25$ dotted and dashed (violet and blue) lines resp.
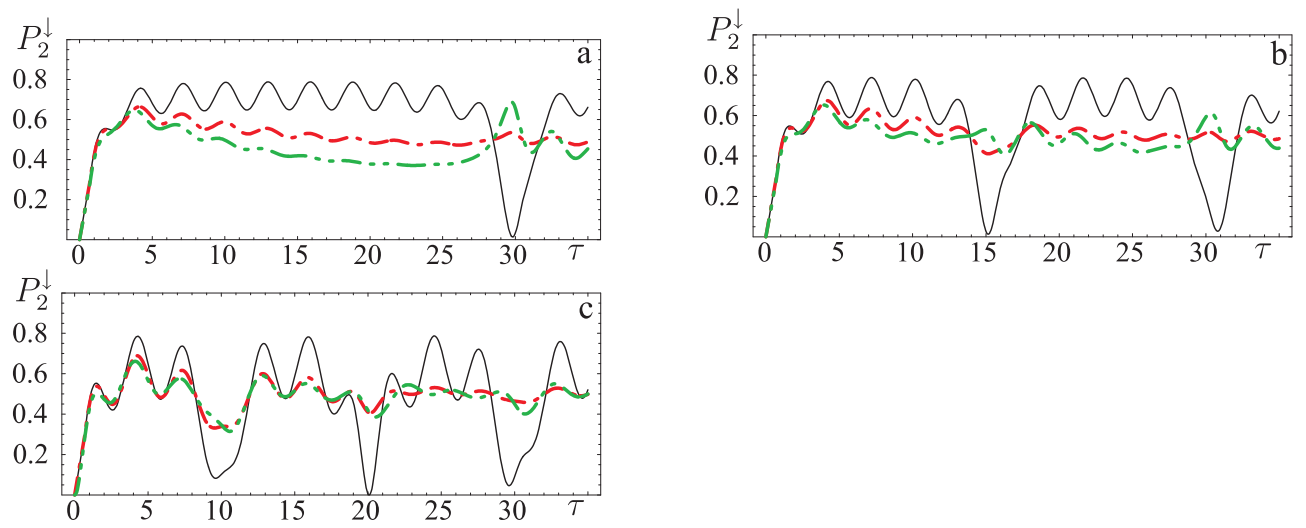

Figure 2. (Color online) (a, c, d) Evolution of $P_{2}^{\downarrow}$ probability at $\delta=\sqrt{3} / 2+0.1$, at dephasing $\left(\Gamma_{\varphi}\right)$ and relaxation $\left(\Gamma_{r}\right)$ rates: $\Gamma_{\varphi}=0.1, \Gamma_{r}=0.05$ dot-dashed (red) line and $\Gamma_{r}=0.2$ double-dot-dashed (green) line; (a) $\omega=0.105$, (b) $\omega=0.205$ and (c) $\omega=0.314$.
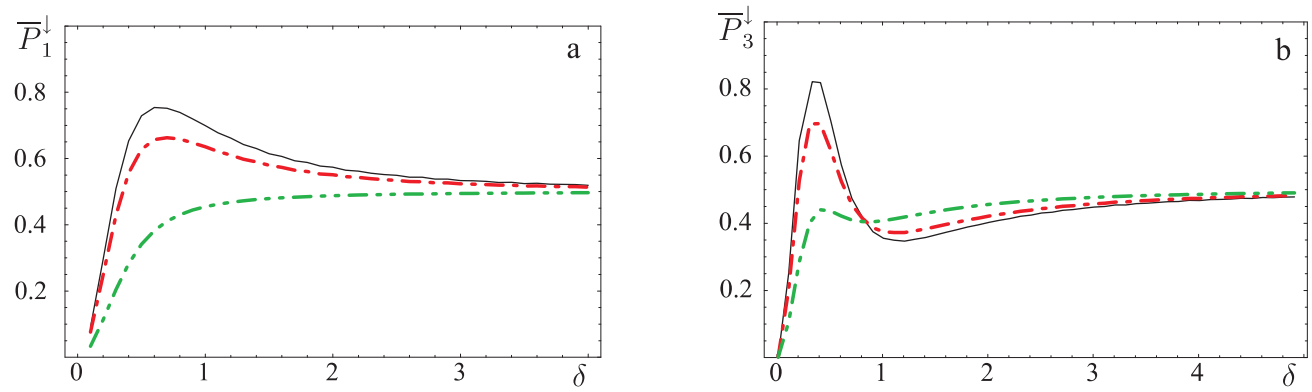

Figure 3. (Color online) $\delta$-dependence of time-averaged probabilities for the closed system solid (black) lines and with dephasing $\left(\Gamma_{\varphi}\right)$ and relaxation $\left(\Gamma_{r}\right)$ rates: $\Gamma_{r}=\Gamma_{\varphi}=0.01$ dot-dashed (red) lines; (a) $\Gamma_{r}=\Gamma_{\varphi}=0.1$ and (b) $\Gamma_{r}=\Gamma_{\varphi}=0.05$ double-dot-dashed (green) lines.

enough to the value $\sqrt{3} / 2$ the minimal value of the probability $P_{1}^{\downarrow}(\tau)$ for $\tau>2$ exceeds 
1/2 (see dashed (blue) and dotted (purple) lines in Fig. 1).

The time-averaged probability as shown in Fig. 3a by solid (black) line exhibits a maximum. Its analytic expression

$$
\overline{P_{1}^{\downarrow}}=2 \delta^{2} \frac{5+4 \delta^{2}}{\left(1+4 \delta^{2}\right)^{2}}
$$

allows us to get the exact position of the maximum which is $25 / 32 \approx 0.78$ at $\delta=\sqrt{5 / 12}$

The result we have just obtained suggests us to consider a more general case $[9,10]$, where function $\epsilon(\tau)$ being periodical depends on three parameters. One of them we fix by re-scaling both time and another parameter (frequency $\omega$ appearing in Eq. (16)) in a way as it has been done above, thus obtaining the field

$$
\epsilon_{2}(\tau)=-1 / 2-\frac{2 \omega^{2}}{b \cos (2 \omega \tau+\varphi)-1 / 2}
$$

where $b^{2}=1 / 4-\omega^{2}>0$. The remaining parameter $\varphi$ can also be eliminated by shifting the time origin so that we put $\varphi=0$ thus reducing external fields to a one parameter $(\omega)$ family. It is important to note that the previous result (12) may be obtained from here at $\varphi=\arctan \omega-\frac{1}{2} \arctan \frac{\omega}{b}$ in the limit $\omega \rightarrow 0$.

According to [10] the analytic expression for $P_{2}^{\downarrow}(\tau)$ reads

$$
\begin{aligned}
P_{2}^{\downarrow}(\tau) & =\frac{4 \delta^{2}}{\theta^{2}} \sin ^{2}\left(\frac{1}{2} \theta \tau\right) \\
& -\frac{4 \delta^{2} b\left[Q-\omega\left(b+b^{2}-\delta^{2}\right) \theta \sin (2 \omega \tau) \sin (\theta \tau)\right]}{\theta^{2}\left(b^{2}+\delta^{2}\right)^{2}(2 b \cos (2 \omega \tau)-1)}
\end{aligned}
$$

where

$$
\begin{aligned}
Q & =b(1+2 b) \theta^{2} \cos ^{2}\left(\frac{1}{2} \theta \tau\right) \sin ^{2}(\omega \tau) \\
& +4 \omega^{2}\left(b-2 \delta^{2}\right) \cos ^{2}(\omega \tau) \sin ^{2}\left(\frac{1}{2} \theta \tau\right) .
\end{aligned}
$$

To show the possibility to control the qubit state by external field (16) we plot function $P_{2}^{\downarrow}(\tau)$ for $\delta$ close to its critical value and for different values of $\omega$. Since the above considered case corresponds to $\omega=0$, we show in Fig. 2a (solid (black) line) its behavior for $\omega=0.105$ which is rather close to zero. During a sufficiently long time interval the probability oscillates between 0.6 and 0.8 after which it falls to zero. The closer gets $\omega$ to zero, the longer this period becomes and the closer to $1 / 2 \omega$ becomes, the more this interval is reduced (see the solid (black) lines in Figs. 2a, 2b and 2c). We have to note that in the limit $\omega \rightarrow 1 / 2(b \rightarrow 0$ in Eq. (16)) function $\epsilon_{2}(\tau)$ tends to a constant value equal $1 / 2$. This signal reproduces the Rabi oscillations with the frequency $2 \sqrt{\delta^{2}+1 / 4}$. Thus, $\omega$ may be considered as a continuously tunable parameter of the external field (16) with the help of which, starting with the usual Rabi oscillations, one may fix the clockwise current direction as long as desirable. We would like to stress that the range of $\delta$, for which the probability exceeds the value $1 / 2$, is rather large, i.e. $0.6<\delta<1.1$. This may facilitate its experimental detection.

Analytic expression for $P_{2}^{\downarrow}(\tau)$ shows us that it represents a complicated superposition of two oscillating functions with frequencies $\theta$ and $2 \omega$. Therefore if these frequencies are close enough to each other one may observe a beating phenomenon (Fig. 4). In this case the oscillations with the small amplitude and the frequency close to the Rabi frequency take place at the background of the oscillations with the 


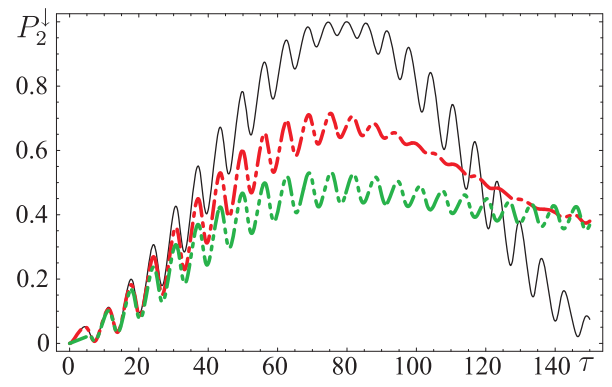

Figure 4. (Color online) Time dependence of clockwise current direction probabilities at $\omega=0.49$ and $\delta=0.1$ solid (black line), at dephasing $\left(\Gamma_{\varphi}\right)$ and relaxation $\left(\Gamma_{r}\right)$ rates: $\Gamma_{r}=\Gamma_{\varphi}=0.01$ dot-dashed (red) line and $\Gamma_{r}=\Gamma_{\varphi}=0.02$ double-dot-dashed (green) line.

amplitude close to 1 and the very small frequency defined by the difference between $2 \omega$ and $\theta$.

Another aspect we would like to emphasize is that this type of the external field is not unique. As we show below, there exist other possibilities for the time dependence of the external field exhibiting a similar feature.

Consider an exactly solvable model with a bit more complicated form of function $\epsilon(\tau)=\epsilon_{3}(\tau)[10]$

$$
\epsilon_{3}(\tau)=-\frac{1}{2}+\frac{6}{Q_{0}}\left(\tau^{4}+6 \tau^{2}-3\right)
$$

where $Q_{0}=\tau^{6}+3 \tau^{4}+27 \tau^{2}+9$. The clockwise current direction probability $P^{\downarrow}(\tau)=$ $P_{3}^{\downarrow}(\tau)$ in this case reads

$$
\begin{aligned}
P_{3}^{\downarrow}(\tau) & =\frac{4\left(\theta^{2}-1\right) \tau^{2}}{\theta^{8} Q_{0}}\left[144\left(1+\tau^{2}\right)+\theta^{4}\left(\tau^{2}+9\right)^{2}-24 \theta^{2}\left(5 \tau^{2}+9\right)\right] \\
& +\frac{\left(\theta^{2}-1\right) Q_{1}}{\theta^{10} Q_{0}}\left[Q_{2} \sin ^{2}\left(\frac{1}{2} \tau \theta\right)+Q_{3} \sin (\tau \theta)\right]
\end{aligned}
$$

where

$$
\begin{aligned}
& \left.\left.Q_{1}=\left[(\theta+1)^{2}-5\right)\right]\left[(\theta-1)^{2}-5\right)\right] \\
& Q_{2}=\theta^{4} Q_{0}+144\left(1+\tau^{2}\right)-12 \theta^{2}\left(5 \tau^{4}+6 \tau^{2}+9\right) \\
& Q_{3}=6 \theta \tau\left[\theta^{2}\left(\tau^{4}+2 \tau^{2}+9\right)-12\left(1+\tau^{2}\right)\right] .
\end{aligned}
$$

We note that this is just the second term in the right hand side of Eq. (18) which is responsible for time oscillations. Therefore if $Q_{1}=0$ the oscillations in the time dependence of the probability disappear and once again it acquires a monotonous character. But this time since $Q_{1}(\theta)$ is a bi-quadratic function, in contrast to the previous case, the clockwise current direction probability turns from an oscillating to monotonous character at two possible values of parameter $\theta, \theta=\sqrt{5} \pm 1$. In these cases the behavior of probabilities $P_{3}^{\downarrow}(\tau)$ and $P_{3}^{\uparrow}(\tau)$ is illustrated in Figs. 5a and 5b (solid (black lines)) respectively. We thus observe for $P_{3}^{\downarrow}(\tau)$ an effect similar to that described above for $P_{1}^{\downarrow}(\tau)$ and, in a sense, the opposite behavior of $P_{3}^{\uparrow}(\tau)$. The existence of the critical value is reflected also by the averaged probability $\bar{P}_{3}^{\downarrow}$ which is plotted in Fig. 3b (solid (black) line). It also has a simple analytic expression

$$
\overline{P_{3}^{\downarrow}}=2 \delta^{2} \frac{13-8 \delta^{2}+16 \delta^{4}}{\left(1+4 \delta^{2}\right)^{2}}
$$



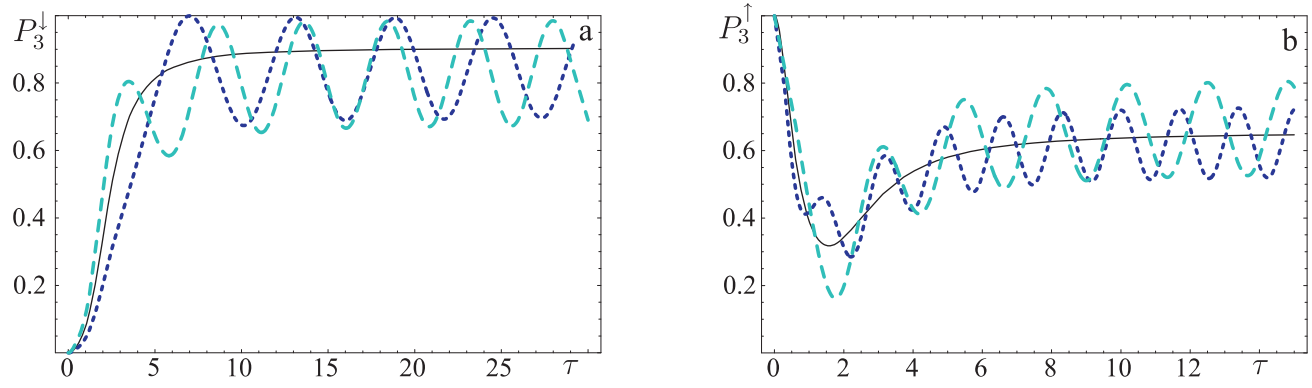

Figure 5. (Color online) Probabilities (a) $P_{3}^{\downarrow}(\tau)$ at $\theta=\sqrt{5}-1$ solid (black) line, $\theta=\sqrt{5}-1.1$ dotted (violet) line and $\theta=\sqrt{5}-0.9$ dashed (blue) line; (b) $P_{3}^{\uparrow}(\tau)$ at $\theta=\sqrt{5}+1$ solid (black) line, $\theta=\sqrt{5}+1.5$ dotted (violet) line and $\theta=\sqrt{5}+0.5$ dashed (blue) line.

with a maximum $\overline{P_{3}^{\downarrow}} \approx 0.91$ at $\delta \approx 0.34$.

The next question we study is how the effect, observing for an idealized closed system, is influenced by a dissipation featuring open quantum systems [16]. To make rough estimations we use a phenomenological approach in the density matrix formalism (see e.g. [17]). In this approach a weak coupling of a system to the environment is described by two parameters, dephasing $\Gamma_{\varphi}$ and relaxation $\Gamma_{r}$ rates. Under the initial condition $2 \rho(0)=I$ with $I$ being identity matrix the elements of the density matrix (cf. [18])

$$
\rho=\frac{1}{2}\left[\begin{array}{cc}
1+Z & X-i Y \\
X+i Y & 1-Z
\end{array}\right]
$$

satisfy the Bloch equations [17]

$$
\begin{aligned}
& \dot{X}=-2 \varepsilon(t) Y-\Gamma_{\varphi} X \\
& \dot{Y}=-2 \delta Z+2 \varepsilon(t) X-\Gamma_{\varphi} Y \\
& \dot{Z}=2 \delta Y-\Gamma_{r}(Z-Z(0)) .
\end{aligned}
$$

Probabilities $P^{\downarrow, \uparrow}$ are defined only by the diagonal entries of the density matrix, $P^{\downarrow, \uparrow}=(1 \mp Z(t)) / 2$.

The relaxation and dephasing effects are shown in Figs. 1-4 by dot-dashed (red) and double-dot-dashed (green) lines. As expected, they disturb the system. The influence of dephasing is more crucial and it should not exceed 5 percent of $\delta$ value for bias (16). We observe an interesting phenomenon concerning the relaxation. When the probability falls to zero the relaxation smoothes this behavior and can even revert it (see green line in Fig. 2a). Thus, in the current case the relaxation may be considered as helping to keep the state of the qubit unchanged.

\section{Concluding remarks}

One of the most popular way to control the qubit state is to drive a two level system with microwave pulses (see e.g. [19]) where, in general, the probability $P^{\downarrow}(\tau)$ displays (possibly controllable) Rabi oscillations. On the other hand it is known that with a specific monochromatic driving force one can "freeze" the state of a two-level system (so called "coherent destruction of tunnelling" [8]). In this paper we report 
on a similar phenomenon but we would like to point out that in principle it can be realized under other physical conditions. This possibility is based on the fact that a magnetic flux with time-dependence like in Eq. (16) may be realized with the help of a superconducting current. Indeed, according to resistively shunted junction model [20] the time dependence of the current going through the junction, which may produce the desirable external flux, reads

$$
I_{s}(t)=I-\frac{I^{2}-I_{c}^{2}}{I+I_{c} \cos \widetilde{\omega} t}, \quad \widetilde{\omega}=\frac{2 e}{\hbar} R \sqrt{I^{2}-I_{c}^{2}}
$$

where $R$ is a resistor parallel to the junction, $I$ is an externally applied constant circuit current and $I_{c}$ is a critical value of the persistent current. The time interval, during which the qubit is in $P_{2}^{\downarrow}$ state, depends on frequency $\omega$ from Eq. (16) which according to Eq. (20) may be continuously tuned with an externally applied current $I$.

\section{Acknowledgments}

The authors would like to thank S. Shevchenko for useful discussions. The work is partially supported by RFBR grant 06-02-16719 and Russia President grant 871.2008.2. V.V.S. acknowledges a partial support from INTAS Fellowship Grant for Young Scientists Nr 06-1000016-6264.

\section{References}

[1] Nakamura Y, Pashkin Yu A and Tsai J S 1999 Nature 398786 Dong B, Horing N J M and Lei X L 2006 Phys. Rev. B 74033303

Zagoskin A M, Ashhab S, Johanson J R and Nori F 2006 Phys. Rev. Lett. 97077001

[2] Nakamura Y, Pashkin Yu A and Tsai J S 2001 Phys. Rev. Lett. 87246601

[3] Mooij J E, Orlando T P, Levitov L, Tian L, C H van der Wal and Lloyd S 1999 Science 285 1036

Orlando T P, Mooij J E, Tian L, C H van der Wal, Levitov L S, Lloyd S and Mazo J J 1999 Phys. Rev. B 6015398

Greenberg Ya S 2003 Phys. Rev. B 68224517

Il'ichev E et al 2003 Phys. Rev. Lett. 91097906

[4] C H van der Wal, Wilhelm F K, Harmans C J P M and Mooij J E 2003 Eur. Phys. J. B 31111

[5] Kim M D 2006 Phys. Rev. B 74184501

[6] Zhang J and Whaley K B 2006 Phys. Rev. A 73022306

[7] Orszag M 2000 Quantum optics (Berlin: Springer-Verlag)

[8] Grossman F, Dittrich T, Jung P and Hänggi P 1991 Phys. Rev. Lett. 67516 Agarwal G S and Harshawardhan W 1994 Phys. Rev. A 50 R4465 Raghavan S, Kenkre V M, Dunlap D H, Bishop A R and Salkola M I 1996 Phys. Rev. A 54 R1781

[9] Bagrov V G, Baldiotti M C, Gitman D M and Shamshutdinova V V 2005 Ann. Phys. 14 No 6 390

[10] Samsonov B F and Shamshutdinova V V 2005 J. Phys. A 384715 Samsonov B F, Shamshutdinova V V and Gitman D M 2005 Cz. J. Phys. 55 No 91173 Shamshutdinova V V, Samsonov B F and Gitman D M 2007 Ann. Phys. (NY) 3221043

[11] Shamshutdinova V V, Kiyko A S, Shevchenko S N, Samsonov B F and Omelyanchouk A N 2006 Preprint quant-ph/0612195

[12] Znojil M, Cannata F, Bagchi B, and Roychoudhury R 2000 Phys. Lett. B 483284

[13] Mostafazadeh A 2002 J. Math. Phys. 43205 Mostafazadeh A 2002 Nucl. Phys. B 640419

[14] Nieto L M, Pecheritsin A A and Samsonov B F 2003 Ann. Phys. (NY) 305151189

[15] B. Mielnik, O. Rosas-Ortiz 2004 J. Phys. A 3710007

[16] Breuer H - P and Petruccione F 2002 The Theory of Open Quantum Systems (Oxford: Oxford University Press)

[17] Blum K 1981 Density Matrix Theory and Applications (New York-London: Plenum Press) 
[18] Shevchenko S N, Kiyko A S, Omelyanchouk A N and Krech W 2005 Fiz. Nizk. Temp. 31 752; Low Temp. Phys. 31569

[19] Saito S, Meno T, Ueda M, Tanaka H, Semba K and Takayanagi H 2006 Phys. Rev. Lett. 96 107001

[20] Likharev K K 1979 Rev. Mod. Phys. 51101

Abrikosov A A 1987 Foundations of Metal Theory (Moscow: Mir) 